\title{
Light-scattering methods for antibiotic sensitivity tests
}

\author{
J MURRAY,* P EVANS, $\dagger$ AND DWL HUKINS*
}

\begin{abstract}
From the ${ }^{*}$ Department of Medical Biophysics, University of Manchester and the $\dagger$ Area Department of Laboratory Medicine, Oldham and District General Hospital, Oldham, Lancs, UK.
\end{abstract}

SUMmARY The total intensity of light scattered in the angular range $30^{\circ}-130^{\circ}$ by a suspension of bacteria can be used as the basis of a method of antibiotic sensitivity testing. A procedure is given in which the bacteria are incubated in the presence of antibiotic, as a test, and in its absence, as a control, for 60 minutes. An incubation time of 90 minutes gives slightly more reliable results. With this increased incubation time it is necessary to measure the intensity of scattered light only at a single fixed angle or, at most, a few angles. Measuring the angular dependence of the scattered intensity does not allow the incubation period to be reduced.

It has been proposed that the angular dependence of the light scattered by a suspension of bacteria could be used as the basis of a rapid method of antibiotic sensitivity testing. ${ }^{1}$ The Differential I light-scattering photometer (Science Spectrum Inc, Santa Barbara, California, USA) was developed for this purpose. ${ }^{2}$ It can be used to plot the logarithm of the intensity of the scattered light against the scattering angle over some required angular range. The result has been called a differential light-scattering (DLS) curve.

The principles involved in sensitivity testing are as follows. Two samples, of identical volume, are taken from a homogeneous suspension of bacteria. Both samples are cultured in a liquid growth medium. Antibiotic is added to the test sample but not to the control sample. When a suitable incubation time has elapsed, DLS curves are obtained from both. A significant difference between the curves indicates that the antibiotic affects the growth or structure of the bacteria.

In principle, the curves can differ in two respects. A difference in scale indicates a difference in the total number of bacteria in each sample-the greater the intensity, the higher the number. Any difference in shape between the curves indicates a change in bacterial morphology. The problem which then arises is to decide whether the difference in scale or shape is significant.

The displacement index, $\mathrm{D}$, has been defined by

$$
\mathrm{D}=\text { Area between curves/Angular range }
$$

and allows for differences in both scale and shape. ${ }^{3}$ Differences in shape alone can be allowed for by

Received for publication 21 February 1980 calculating the area between the two curves and dividing by the angular range, as before, after they have been scaled together so as to have the same average values over their angular range. The result is called the morphological index, $\mathbf{M}$, and is combined with $\mathrm{D}$ to give a total score, $\mathrm{S}$, defined by

$$
\mathbf{S}=\mathbf{D}+3 \mathbf{M}
$$

for the sensitivity tests. ${ }^{3}$ (The factor of 3 was chosen to obtain equal contributions from the $\mathrm{D}$ and $3 \mathrm{M}$ terms after the bacteria had been incubated for 90 minutes. ${ }^{3}$ )

The intensity of the light scattered at a single fixed angle had already been used for sensitivity testing. ${ }^{4}$ But this technique could yield incorrect results if the effect of the antibiotic were greatly to change the shape of the DLS curve. ${ }^{5}$ This effect will be considered in greater detail later (see Results).

Our experiments were designed to investigate these various methods of antibiotic sensitivity testing using the Differential I photometer. We find that the morphological term, $M$, is of little value as the total score, $S$, provides no more significant information than the displacement index, D. Furthermore, measurements at a fixed angle are not often misleading. The occasional problems that arise could be overcome with a simpler instrument than the Differential $\mathrm{I}$. We also find that a suitable incubation time is 60 or 90 minutes, depending on the exact method chosen, and that the results are not significantly influenced by noise in the DLS curves.

In these experiments the light-scattering results were compared with the results of agar plate sensitivity tests. Preliminary experiments showed that the light-scattering indices, D and S, were not strongly correlated with the zone diameters in the 
plate tests. Therefore these tests were used to decide whether bacteria were sensitive or insensitive to a given antibiotic. It was necessary to find a boundary level for the value of each index above which the bacteria would be judged to be sensitive to the antibiotic in the light-scattering tests. The number of times when agar plate and light-scattering tests disagreed was plotted against trial boundary levels; a minimum in the resulting graph indicated the optimum level. This procedure was repeated for $\mathrm{D}, \mathrm{S}$, and the results obtained from single angle measurements after three different incubation periods. The entire set of analyses was repeated with cubic spline representations of the curves, which correspond to noise-free DLS results, ${ }^{6}$ in order to assess the effects of noise on the results.

\section{Material and methods}

\section{BACTERIA AND ANTIBIOTICS}

Two hundred samples of bacteria were used in the tests. These were chosen as representative of a wide range of clinical samples obtained for routine testing.

Antibiotics were used in the form of impregnated paper discs (Mast Laboratories Ltd, Liverpool, UK) and were stored at $4{ }^{\circ} \mathrm{C}$. The antibiotics tested for use against the different genera of bacteria are listed in Table 1.

\begin{tabular}{ll}
$\begin{array}{l}\text { Table 1 } \\
\text { bacteria }\end{array}$ & Antibiotics used against different types of \\
\hline Bacteria & Antibiotic \\
\hline $\begin{array}{c}\text { Gram-positive } \\
\text { Staphylococcus }\end{array}$ & $\begin{array}{c}\text { Penicillin, methicillin, erythromycin, } \\
\text { lincomycin, fucin }\end{array}$ \\
Streptococcus & $\begin{array}{c}\text { Penicillin, erythromycin, lincomycin, } \\
\text { tetracycline }\end{array}$ \\
$\begin{array}{c}\text { Gram-negative } \\
\text { Klebsiella and } \\
\text { Escherichia } \\
\text { Proteus }\end{array}$ & $\begin{array}{c}\text { Ampicillin, tetracycline, mecillinam, } \\
\text { streptomycin, gentamicin } \\
\text { Ampicillin, carbenicillin, streptomycin, } \\
\text { gentamicin }\end{array}$ \\
Pseudomonas & Streptomycin, carbenicillin, gentamicin \\
\hline
\end{tabular}

\section{AGAR PLATE TESTS}

Plate sensitivity tests were carried out by a method based on those described previously. ${ }^{7} 8$ Standard organisms were strains of Staphylococcus aureus (when testing Gram-positive spheres), Escherichia coli (when testing Enterobacteriaceae), and Pseudomonas pyocyanea (when testing the genus Pseudomonas). Problems encountered in this technique are discussed by Stokes and Waterworth. ${ }^{8}$

Zone diameters were measured, to the nearest millimetre, using callipers. Bacteria were classified as sensitive if the zone diameter exceeded $18 \mathrm{~mm}$.

\section{LIGHT-SCATTERING TESTS}

All incubations referred to below were at $37^{\circ} \mathrm{C}$, and when a medium was prewarmed it was always to this same temperature. Bacteria were first incubated, usually overnight, on blood agar plates. A few colonies were then transferred to a small bottle containing prewarmed brain-heart infusion $(4 \mathrm{ml}$; full strength), using a flamed wire loop, and incubated for 30 minutes. After incubation the bottle was shaken, to ensure an even distribution of bacteria, and a sample $(0.4 \mathrm{ml})$ was added to each of three Berkman-Schoefer cuvettes. ${ }^{9}$ Each cuvette contained prewarmed brain-heart infusion $(15 \mathrm{ml}$; $1 / 3$ strength).

Two cuvettes were used for tests and contained antibiotic, a different one in each. The antibiotics used are given in Table 1 and the concentration in the cuvettes in Table 2 . In some cases, preliminary tests had to be carried out with standard organisms to ascertain the best concentration to use, but for most antibiotics suitable concentrations were already available. ${ }^{10}$ The third cuvette was used as a control for both tests and so contained no antibiotic.

Table 2 Concentrations of various antibiotics used in tests

\begin{tabular}{ll}
\hline Antibiotic & Concentration \\
\hline Ampicillin & $2.0 \mu \mathrm{g} / \mathrm{ml}$ \\
Gentamicin & $0.5 \mu \mathrm{g} / \mathrm{ml}$ \\
Streptomycin & $1.0 \mu \mathrm{g} / \mathrm{ml}$ \\
Tetracycline & $0.25 \mu \mathrm{g} / \mathrm{ml}$ \\
Lincomycin & $0.25 \mu \mathrm{g} / \mathrm{ml}$ \\
Erythromycin & $0.06 \mu \mathrm{g} / \mathrm{ml}$ \\
Penicillin & $0.1 \mathrm{U}$ \\
Fucin & $1.5 \mu \mathrm{g} / \mathrm{ml}$ \\
Methicillin & $1.5 \mu \mathrm{g} / \mathrm{ml}$ \\
Carbenicillin & $5 \mu \mathrm{g} / \mathrm{ml}$ \\
Mecillinam & $1 \mu \mathrm{gl} / \mathrm{ml}$ \\
\hline
\end{tabular}

Cuvettes were incubated and DLS curves recorded after periods of 30,60 , and 90 minutes. Before each curve was recorded the cuvette was allowed to rest in the chamber of the Differential I for about 1 minute. This procedure greatly reduced the noise level in the curves, probably because large particles settled to the bottom of the cuvette where they could not intercept the light beam. Not more than 20 tests could be performed during a 5-hour period. But this number could presumably be greatly increased by using the Differential III light-scattering photometer (Science Spectrum Inc, Santa Barbara, California, USA) which is an updated version of the Differential I, incorporating automatic sample changing. ${ }^{10}$

ANALYSIS OF DLS CURVES

The logarithm of the scattered intensity for the angular range $30^{\circ}-130^{\circ}$ was punched on to paper 
tape, at $1^{\circ}$ intervals, for analysis. For each sensitivity test the displacement index, D, and total score, S, were calculated. ${ }^{3}$ Also the difference between the logarithm of the intensity scattered at a fixed angle in a test and its control was calculated. Differences were calculated for angles of $30^{\circ}, 60^{\circ}, 90^{\circ}$, and $120^{\circ}$. All calculations were performed for the results obtained after each incubation period of 30,60 , and 90 minutes.

Noise-free curves were obtained by fitting a cubic spline to all experimental curves. ${ }^{6}$ Every calculation was repeated using these cubic spline representations in place of the curves obtained experimentally.

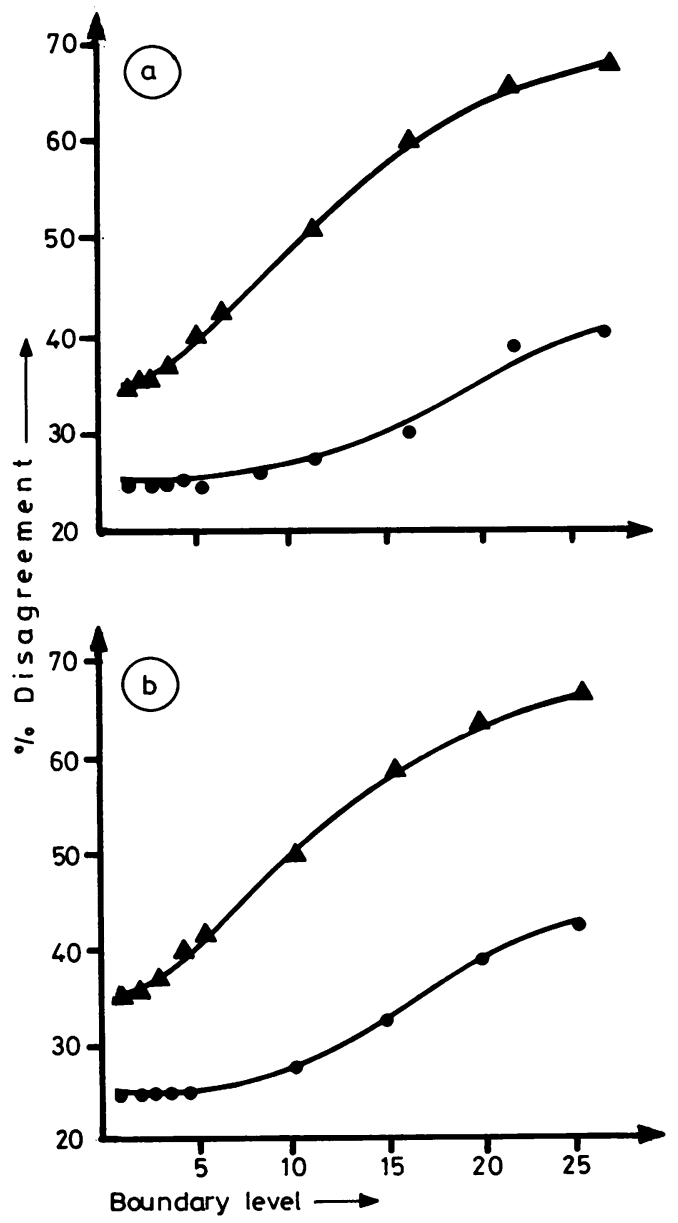

Fig. 1 Graph of variation in percentage disagreement between agar plate results and displacement index, $D$ $(\Delta)$, and the total score, $S(\mathbf{O})$, after 30 minutes' incubation, against trial boundary level. The experimental DLS curves were used for (a) and their cubic spline representations for (b).
Results

Figure 1 shows that 30 minutes is an insufficient incubation period. It did not matter whether the experimental DLS curve or its cubic spline representation was chosen, or whether $\mathbf{D}$ and $\mathrm{S}$ was chosen as the index. The graphs of disagreement between agar plate and DLS tests against trial boundary levels showed no minima. Thus, there was no optimum boundary level for the DLS tests.

After an incubation time of 60 minutes the DLS results are more useful; once again the simple index, $\mathrm{D}$, proved useful as the total score, $\mathrm{S}$, that is incorporating morphological information, made little difference to the outcome of the tests. In the curves shown in Fig. 2, the percentage disagreement between

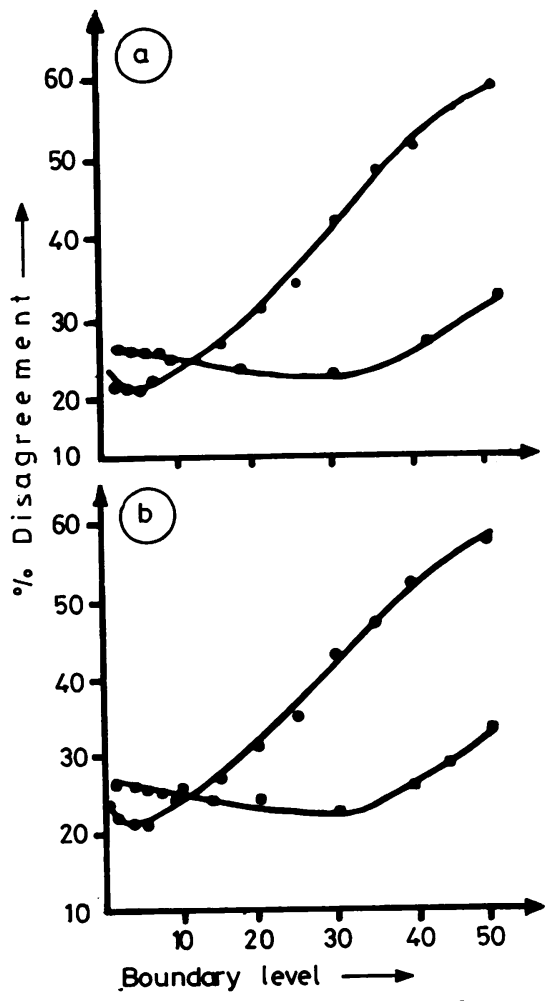

Fig. 2 Graph of variation in percentage disagreement between agar plate results and displacement index, $D(O)$, and the total score, $S(\square)$, after 60 minutes' incubation, against trial boundary level. The experimental DLS curves were used for (a) and their cubic spline representations for $(\mathrm{b})$.

agar plate and DLS test results is lower than in Figure 1. At the minima, the positions of which represent optimum DLS boundary levels, the dis- 
agreement is just over $20 \%$. In fact the disagreement is somewhat lower for $\mathbf{D}$ than for $\mathbf{S}$. Once again the effect of replacing experimental curves by their cubic spline representations is negligible.

When the incubation time was increased to 90 minutes the agreement between DLS and agar plate tests was even better; but, as before, both $\mathrm{D}$ and $\mathrm{S}$ terms were equally useful, and the cubic spline representations were no more useful than the experimental DLS curves. Figure 3 shows that in all cases the level of disagreement fell to around $17 \%$ when the optimum boundary level was chosen.

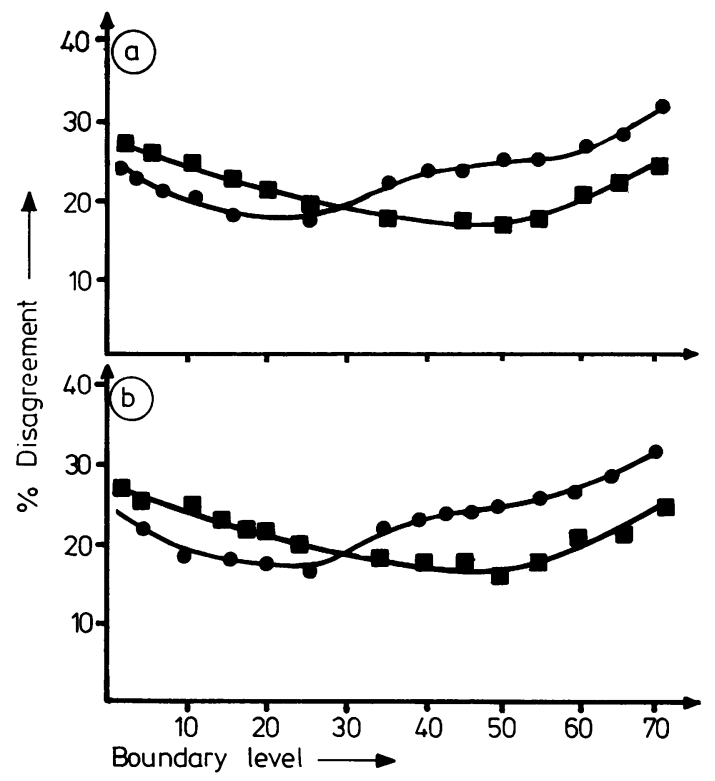

Fig. 3 Graph of variation in percentage disagreement between agar plate results and displacement index, $D(O)$, and the total score, $S(\square)$, after 90 minutes, incubation, against trial boundary level. The experimental DLS curves were used for (a) and their cubic spline representations for (b).

Why does $\mathbf{S}$ contain no more useful information than D? Figure 4 compares test and control DLS curves obtained for the action of lincomycin on a sensitive strain of Staph. aureus; it is apparent that the antibiotic causes the peak positions in the DLS curves to shift. The shaded area between the test and control curves in this figure is proportional to $D$. In Fig. 5 these curves have been scaled together so that the shaded area between them is now proportional to the morphological index, M. Comparison of the two figures shows that $\mathbf{D}$ is always much greater than $\mathbf{M}$, so that, when the two are combined to compute $S$, the morphological index has little effect. $D$ and $S$ then convey essentially the same information.

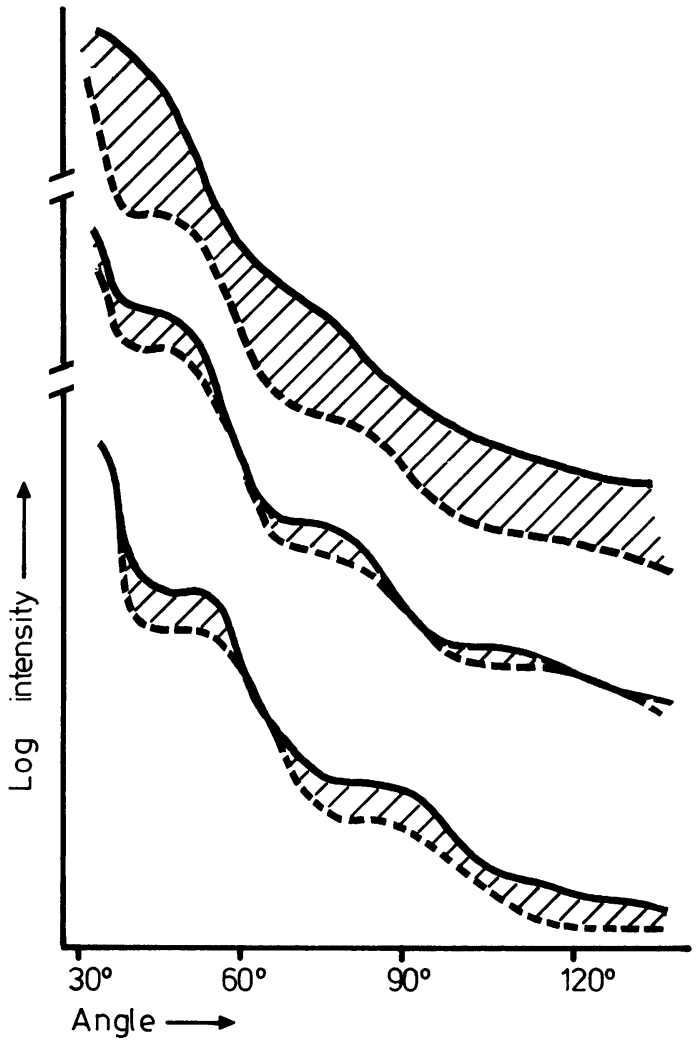

Fig. 4 DLS curves from a sensitive strain of Staph. aureus in the presence of lincomycin (- - ) after incubation times of 30,60 , and 90 minutes. The hatched area between test and control (__ to displacement index, D, multiplied by the angular range.

Figure 6 compares the results of the $60^{\circ}$ single-angle tests with the agar plate results. Results obtained at $30^{\circ}, 90^{\circ}$, and $120^{\circ}$ were very similar, indicating that the chosen angle has little effect on the results of these tests. After an incubation time of only 30 minutes the disagreement was very high at around $40 \%$. By 60 minutes it had dropped to around $27 \%$. After 90 minutes the percentage disagreement had again fallen, this time to just below $20 \%$. There was no noticeable effect resulting from the replacement of the experimental curves by their cubic spline representations.

Figure 7 shows why the problems anticipated by Stull ${ }^{5}$ are not important after an incubation time of 90 minutes. This figure compares test and control DLS curves obtained for the action of tetracycline on a sensitive strain of Staph. aureus. Because one effect of the antibiotic is to shift the peaks in the 


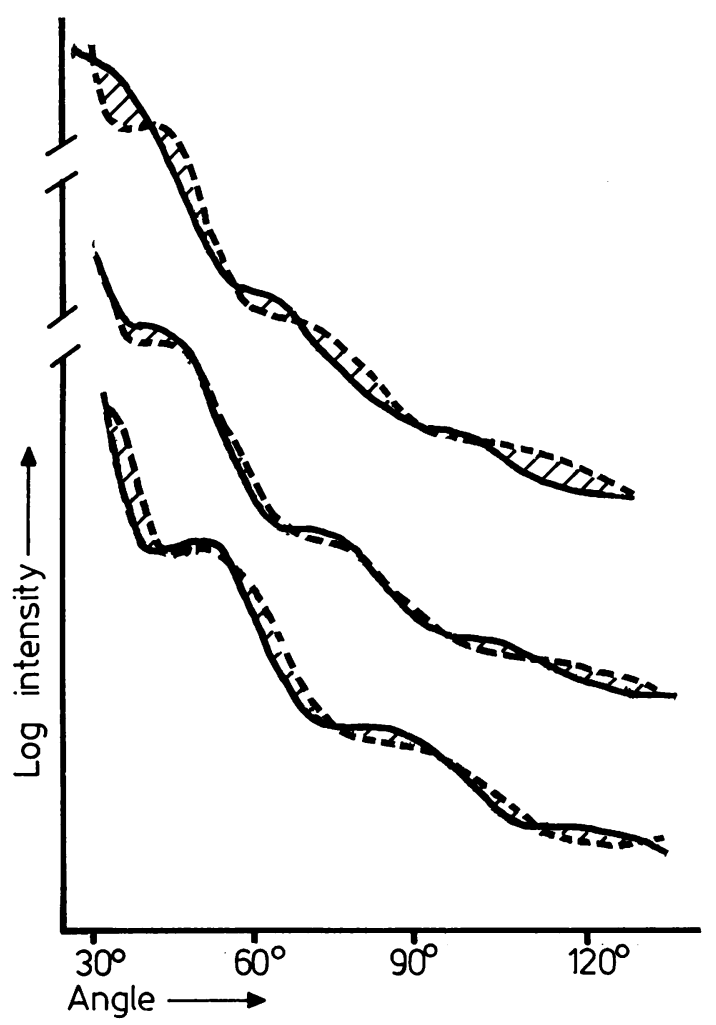

Fig. 5 The same DLS curves as in Fig. 4 but with test and control curves scaled together so that the hatched area between them is equal to the morphological index, $M$, multiplied by the angular range.

DLS curve, a single-angle measurement at $60^{\circ}$ would yield an 'insensitive' result because the test scatters no less light around this angle than the control after 30 minutes' incubation. However, in the total range $30^{\circ}-130^{\circ}$, the test specimen clearly scatters less light. Thus, the single result is misleading. But after 90 minutes' incubation the effect of antibiotic is more marked and less light is scattered at a single angle of $60^{\circ}$, reflecting the behaviour throughout the angular range. Thus, the single angle measurement is no longer misleading.

\section{Discussion}

It is clear that, in our experience, the total score, $\mathbf{S}$, introduced by Wyatt and his collaborators ${ }^{3}$ is of little more value than their simple displacement index, D, in sensitivity tests. An incubation period of 60 minutes would be adequate, but 90 minutes would lead to slightly more reliable results. The simple index is proportional to the difference in the

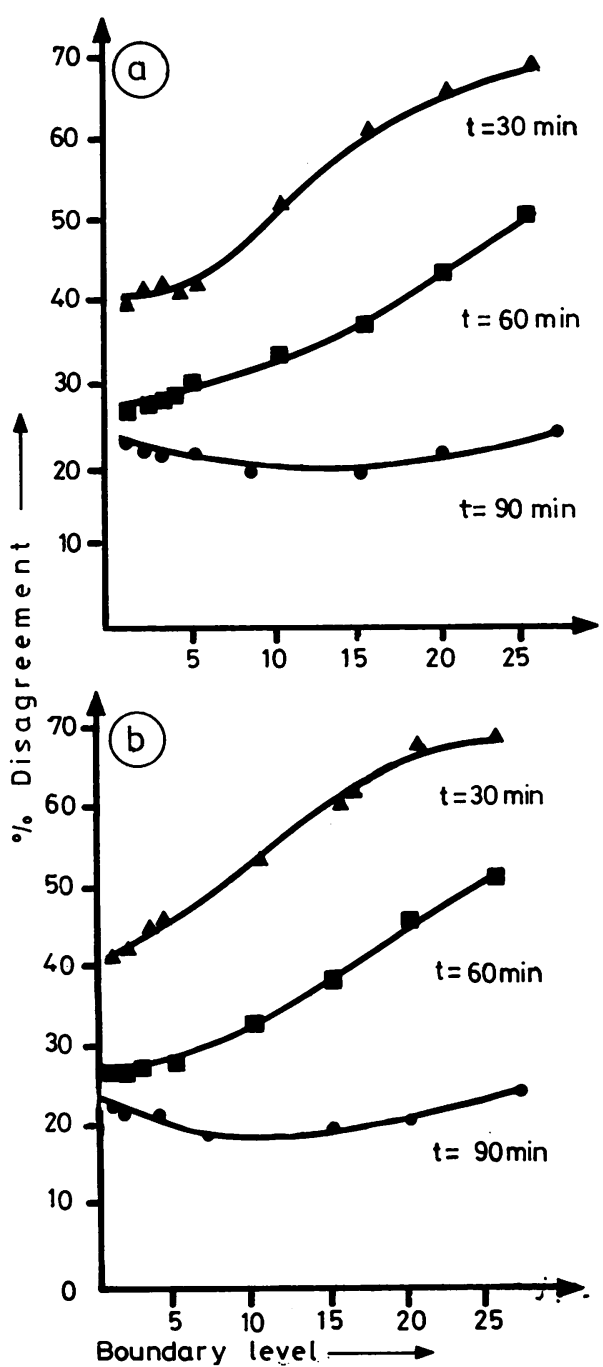

Fig. 6 Graph of variation in percentage disagreement between agar plate results and results based on lightscattering at a single angle $\left(60^{\circ}\right)$ against trial boundary level, after incubation times of 30,60, and 90 minutes. The experimental DLS curves were used for (a) and their cubic spline representations for (b).

logarithm of the total intensity of light scattered, in the chosen angular range, by the test and control samples. Figure 4 makes the same point pictorially.

Consequently, there is no need to measure the angular dependence of the light scattered. The total scatter in the required range for the test and control would be sufficient to calculate $D$. This total scatter could be measured with a conceptually simpler instrument than the Differential I. With an incuba- 


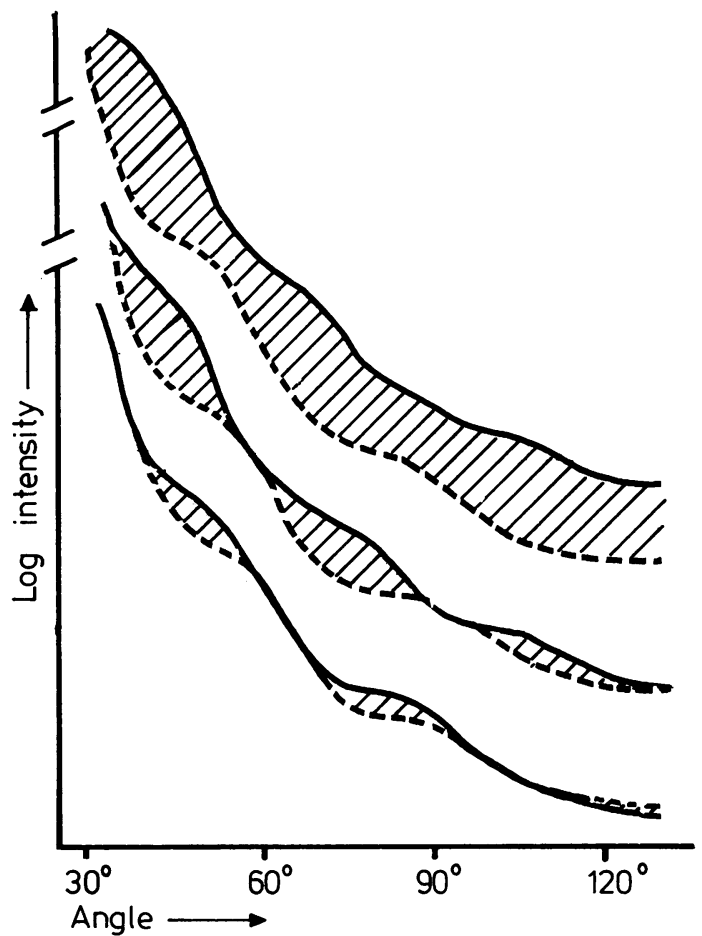

Fig. 7 Control (—) and test (- - - DLS curves obtained for the action of tetracycline on a sensitive strain of Staph. aureus after incubation times of 30,60 , and 90 minutes.

tion time of 90 minutes an even simpler instrument which made a single-angle measurement would be adequate, although it might be preferable to make a few such measurements simultaneously and add the results. (Of course, if sufficient single-angle measurements are made the difference between the test and control sums will be proportional to D.) In practice, the simpler instruments which are used for antibiotic sensitivity testing are more complex than a simple nephelometer and are comparable in price to the Differential I.

How can we account for the $20 \%$ disagreement between DLS and agar plate tests? Some disagreement is inevitable when sharp, yet arbitrary, boundaries between 'sensitive' and 'resistant' are defined. In about one half of the cases of disagreement one method resulted in a score that was at the bottom end of the 'sensitive' scale and the other in a score at the top end of 'resistant'. We classify these as minor discrepancies.

There are three reasons for major discrepancies. A few cases were found where the agar plate method gave a 'sensitive' result, yet growth of the bacteria was not affected by the antibiotic in a cuvette. A probable cause is an inactive antibiotic disc; in the agar plate tests this problem was overcome by placing the disc between test and standard organisms. A strictly analogous method is not possible in the DLS tests if antibiotic is added in the form of impregnated discs. But the problem could be overcome by not using discs and regularly testing batches of antibiotic against standard organisms. The second major cause of discrepancy was failure of the bacteria to grow. Here the DLS curves were characterised by a featureless, noisy appearance, and the intensity scattered by the control was, of course, much less than usual. This latter characteristic could be detected in a completely automated system and the test could be repeated. Finally, it must be remembered that the agar plate method can give a false result. In a trial, 114 errors were noted in 3386 tests. ${ }^{11}$

The advantage of the DLS curve is that its form allows a reasonably intelligent guess as to the identity of the organism causing infection. This advantage is of obvious importance in the examination of cerebrospinal fluid from patients admitted with suspected meningitis. Identification of bacteria in this way has been proposed as another application of the DLS technique. ${ }^{12}$ Furthermore, light-scattering photometers, such as the Differential I, are valuable research instruments for examining the structures of bacteria or indeed of any other particles of comparable size.

We thank Professor BR Pullan for encouragement and the North Western Regional Health Authority for financial support.

\section{References}

${ }^{1}$ Berkman RM, Wyatt PJ, Phillips DT. Rapid detection of penicillin sensitivity in Staphylococcus aureus. Nature 1970;228:458-60.

2 Phillips DT. Evolution of a light scattering photometer. BioScience 1971 ;21:865-7.

${ }^{3}$ Wyatt PJ, Phillips DT, Allen EH. Laser light scattering bioassay for veterinary drug residues in food producing animals. 1. Dose-response results for milk, serum, urine and bile. $J$ Agric Food Chem 1976;24:984-8.

${ }^{4}$ Kavanagh F. Turbidimetric assays: The antibiotic doseresponse line. Appl Microbiol 1968;16:777-80.

${ }^{5}$ Stull VR. Clinical laboratory use of differential light scattering. I. Antibiotic susceptibility testing. Clin Chem 1973;19:883-90.

- Murray J, Hukins DWL, Evans P. Application of Mie theory and cubic splines to the representation of light scattering patterns from bacteria in the logarithmic growth phase. Phys Med Biol 1979;24:408-15.

${ }^{7}$ Stokes EJ. Quality control in diagnostic bacteriology. Proc $R$ Soc Med 1968;61:457-63.

${ }^{8}$ Stokes EJ, Waterworth PM. Antibiotic sensitivity tests by diffusion methods. Association of Clinical Pathologists Broadsheet 55, 1972.

9 Berkman RM, Schoefer PB. US Patent No. 3701620, 1972. 
10 Wyatt PJ. Differential Light Scattering, Antibiotic Susceptibility Testing and the Differential III System. Santa Barbara: Science Spectrum, 1974.

11 Association of Clinical Pathologists sensitivity test trial. $J$ Clin Pathol 1965;18:1-5.

12 Wyatt PJ. Identification of bacteria by differential light scattering. Nature 1969;221:1257-8.

Requests for reprints to: Dr DWL Hukins, Department of Medical Biophysics, University of Manchester, Stopford Building, Oxford Road, Manchester M13 9PT, UK.

\section{THE SEPTEMBER 1980 ISSUE CONTAINS THE FOLLOWING PAPERS}

Role of intestinal metaplasia in the histogenesis of gastric carcinoma JR JASS

Evaluation of endoscopic brush and biopsy touch smear cytology and biopsy histology in the diagnosis of carcinoma of the lower oesophagus and cardia JENNIFER A YOUNG, HELENA E HUGHES, AND FD LEE

A new cryopathic syndrome M CAUDRON, C BIEVA E LAURENT, F DE MEUTER, WW FEREMANS, P LEBAS, AND CL MALMENDIER

Perineal rhabdomyosarcoma in a newborn child: pathological and biochemical studies with emphasis on contractile proteins HF HILDEBRAND, I KRIVOSIC, X GRANDIER-VAZEILLE, D TETAERT, AND G BISERTE

Reporting practices of microbiology laboratories VP ACKERMAN, RC PRITCHARD, DJ GROOT OBBINK, R BRADBURY, AND A LEE

Serological diagnosis of Mycoplasma pneumoniae infection by enzyme immunoassay SM RÄISÄNEN, JI SUNI, AND PO LEINIKKI

Enzyme-linked immunosorbent assay for the serodiagnosis of toxoplasmosis D MILATOVIĆ AND I BRAVENY

Rubella antibody determination from heparinised finger-tip blood by single radial haemolysis and enzyme immunoassay ANTTI VAHERI, PERTTI VÄÄNÄNEN, EEVA-MARJATTA SALONEN, AND JUKKA SUNI

Arizona hinshawii bacteraemia and meningitis in a child: a case report REBECCA ICHORD, I BROOK, AND G CONTRONI
Hepatitis B carrier state produced by a blood transfusion J NAGINGTON, TG WREGHITT, RS TEDDER, AND DS DANE

Antibiotic sensitivities of urinary pathogens, 1971-8 RN GRÜNEBERG

Pathology measurements closer to the patient? K WIENER

Air bubbles and temperature effect on blood gas analysis G MADIEDO, R SCIACCA, AND L HAUSE

Hyperthyroidism with normal serum total T4, T3, and thyroid binding protein concentration JF CARO, NG SLOANE, AND JA GLENNON

Serum immunoreactive trypsin concentrations in diabetic children ANNE MOFFAT, $V$ MARKS, AND DR GAMBLE

Anti-actin antibodies revealed by counter-immunoelectrophoresis $\mathrm{H}$ DIEDERICHSEN AND KIRSTEN RIISOM

Changes in the molecular size distribution and posttransfusion survival of hydroxyethyl starch $350 / 0 \cdot 60$ as influenced by a lower degree of hydroxyethylation: a study in normal man JM MISHLER, CR RICKETTS, AND EJ PARKHOUSE

Haemagglutination kinetics using a continuous-flow system Ph ROUGER, P GANE, AND Ch SALMON

Septic shock-ACP Symposium

Copies are still available and may be obtained from the PUBLISHING MANAGER, BRITISH MEDICAL ASSOCIATION, TAVISTOCK SQUARE, LONDON WC1H 9JR, price $£ 3.00$, including postage 